\title{
Performance Comparison between Ant Algorithm and Modified Ant Algorithm
}

\author{
Shaveta Malik ${ }^{\#}$

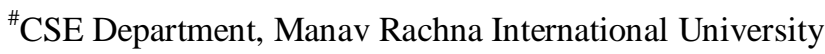 \\ Faridabad, Haryana-121001 INDIA \\ shavetamalik687@gmail.com
}

\begin{abstract}
This paper gives a brief about two of the metaheuristic techniques that are used to find best among the optimal solutions for complex problems like travelling salesman problem, Quadratic problem. Both of these techniques are based on the natural phenomenon of ant. Ant algorithm find good path but due to some short comings of it, this algorithm is not able to give best out of the good or optimal solutions, but modified ant algorithm which is based on probability finds out the best among the optimal paths We will also see that the modified ant algorithm can obtain less number of hops which helps us to get the best solution to typical problems.
\end{abstract}

Keywords - Ant Algorithm, Modified Ant algorithm, Travelling Salesman Problem, Quadratic problem, Ant System

\section{INTRODUCTION}

Both meta-heuristic techniques i.e. ant algorithm and modified ant algorithm are based on of the natural phenomenon that ants take the shortest path as and when they move out from their nest in search of their food and vice-versa. The ant algorithm can be used to traverse the shortest path for the complex problem like travelling salesman. When probability comes into picture it makes work of selecting the shortest or the best path from the good ones a bit easy. We have simulated the nodes for Travelling Salesman problem using ant algorithm and modified Ant algorithm. Study below shows us that the modified ant algorithm gives lesser number of hopes than Ant algorithm at the same connection time. Modified ant algorithm technique chooses the best among all good paths that are available. As a part of study we will see that the average number of hops at connection time after changing the probability by the formula of ant algorithm gives lesser number of hops and hence gives the better option for selecting the shortest path. Experimentation will show that modified ant algorithm is a promising optimization technique for solving complex combinatorial optimization problems like TSP. Modified ant algorithm makes use of the probability theory to get the maximum pheromone where ant can go and take their food gives us the best path among the goods.

In ant colony optimization algorithms, all ants begin to generate the same result. This situation is named as stagnation behaviour, because after a point algorithm stops to generate alternative solutions. The reason of this situation is after a certain number of iterations, ants start generating the same solutions because pheromone amount intensifies in some points and the difference between pheromone concentrations on paths become very huge.

At this point modified ant algorithm comes into picture and after calculating the best probability of the pheromone the optimal path is taken out from the simulations results related to ant algorithm and modified ant algorithm.

\section{BACKGROUND}

Social insects like ants, bees or termites are generally conceived as simple, non-intelligent animals however, collectively they exhibit impressive problem solving skills. Inspired by these insects research in the past decade has led to some fascinating progress in the field of natural algorithms. In the real world, ants (initially) wander randomly, and upon finding food return to their colony while laying down pheromone trails. If other ants find such a path, they are likely not to keep travelling at random, but to instead follow the trail, returning and reinforcing it if they eventually find food. Over time, however, the pheromone trail starts to evaporate, thus reducing its attractive strength. The more time it takes for an ant to travel down the path and back again, the more time the pheromones have to evaporate. A short path, by comparison, gets marched over faster, and thus the pheromone density remains high as it is laid on the path as fast as it can evaporate. Pheromone evaporation has also the advantage of avoiding the convergence to a locally optimal solution.

If there were no evaporation at all, the paths chosen by the first ants would tend to be excessively attractive to the following ones. In that case, the exploration of the solution space would be constrained. Thus, when one ant finds a good (i.e. shortest) path from the colony to a food source, other ants are more likely to follow that path, and positive feedback eventually leads all the ants following a single path. The idea of the ant colony algorithm is to mimic this behaviour with "simulated ants" walking around the graph representing the problem to solve.

In the existing ant algorithm ant will travel to the node whose probability is higher, out of these ant used to select one path and use to travel to all the nodes with high probabilities only. In this process ant generally doesn't look at the other possible solution or paths available for her to traverse and in this way sometime ant used to miss the best among the good paths. Modified ant algorithm overcomes this problem via looking at the all possible solutions and calculating the shortest path to cover all the paths.

\section{FORMULA USED}

Ant system algorithm can be described as in following steps:

(a) Parameters:

Parameters that are used to evaluate and analyse the performance of ant algorithm mentioned below:

- $\quad$ Processed node probability

- Update probability 
- $\quad$ Ratio of proceed node probability

The formula of finding the probability and modification of probability:-

$$
\begin{aligned}
& \mathrm{r}=\sum \mathrm{pi} / 100 \mathrm{i}=1-\mathrm{n} \\
& \mathrm{pu}=\left((\mathrm{pi}+100)+\left(\sum \mathrm{p}-\mathrm{pi}\right)\right) * \mathrm{r} \\
& \mathrm{r} \rightarrow>\text { ratio } \\
& \text { pi } \rightarrow \text { processed node prob. } \\
& \mathrm{p}->\text { prob. Of nodes before processing } \\
& \text { pu } \rightarrow \text { Updated Probability. }
\end{aligned}
$$

(i) Calculation of probability:-

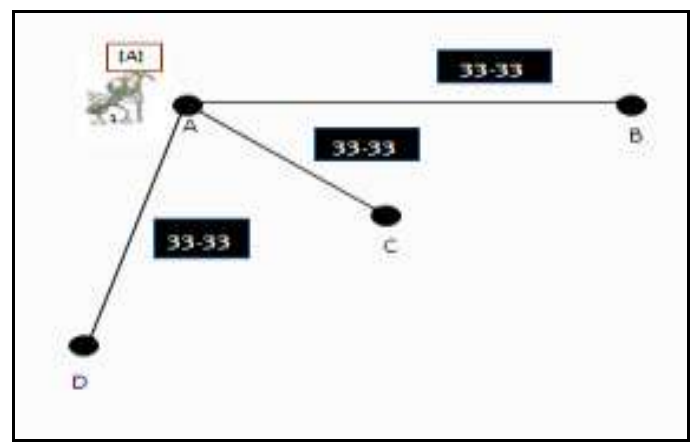

Fig 1 Equal probability at all nodes

(i) How probability is calculated?

The above formula is used to calculate the probability

1) Initialize

Node 2 was the destination (probability $1=100 \%$ ), divide $100 \%$ into three paths as shown in the fig 1 above. Probability of all the three paths i.e. A to B is $33.33 \%$, A to C is $33.33 \%$ and $\mathrm{A}$ to $\mathrm{D}$ is $33.33 \%$

In which $\mathrm{m}$ ants are positioned on $\mathrm{n}$ towns. The starting town and the nodes allowed to visit are initialized for each ant.

2) Iteration

//traverse all nodes, and come back to the starting nodes.

\{The ant moves to next town according to the highest probability in second iteration $\}$

3) It takes 1 hop to get its destination

Divide 1 hop by 100:100\%

Add 100 to the probability value of node 2(currently 33.333 ) $=133.333$.

$100+33.333=133.333 \%$

4) Add the values of the other nodes to 133.3333 $(133.3333+33.3333+33.3333)=200$ (approximately)
Calculate the ratio: ratio $=100 / 200=0.5$

Vol. 1, No. 4, October 2010

Set the probability of the node to its current value multiplied by the ratio

Node 2: $133.3333 *$ ratio $(0.5)=66.6666 \%$

Node 3: $33.3333 *$ ratio $(0.5)=16.6666 \%$

Node $4: 33.3333 *$ ratio $(0.5)=16.6666 \%$ Node 2

$(66.6666 \%)+$ Node $3(16.6666 \%)+$

Node $4(16.6666 \%)=99.9999 \%$

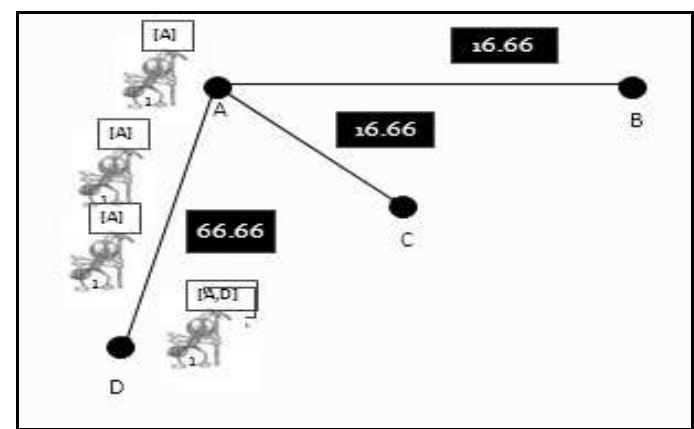

Fig 2 Shows the after calculating probability of pheromone Node A has highest probability

To improve the above algorithm traverse all the nodes to select the path in first iteration and in second iteration select the second path and traverse all the path and find the good paths and after that among the good paths select the best path and modified algorithm will be better as compare to the simple ant algorithm and number of hops will be less in modified algorithm.

\section{EXPERIMENTATION}

A. Ant algorithm

In this section, the simulation results on 100 nodes are shown.

1) At first simulation in ant colony algorithm

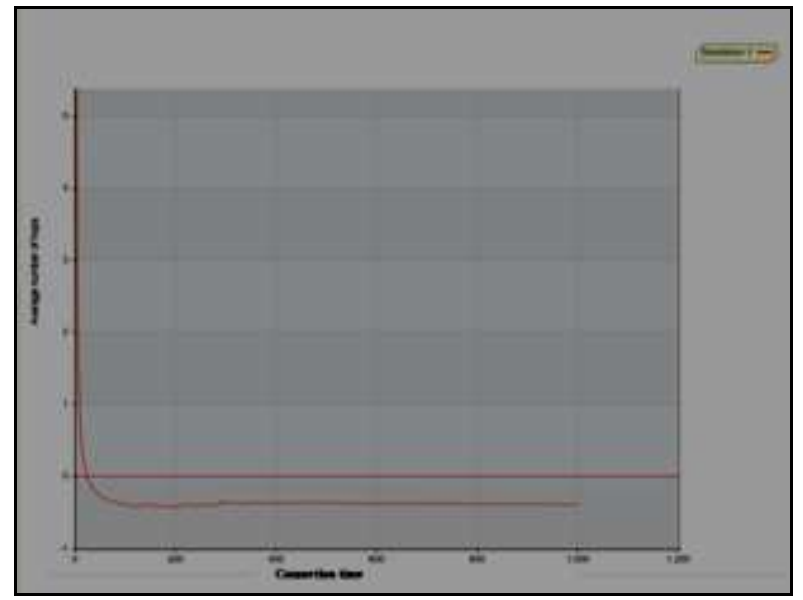

Fig. 3 Average number of hops vs Connection time 
Fig 3 shows the average number of hops on connection time with modification of the probability in ant algorithm and number of hops will change according to the modification of the probability in 100 nodes.

2) At second simulation in ant colony algorithm

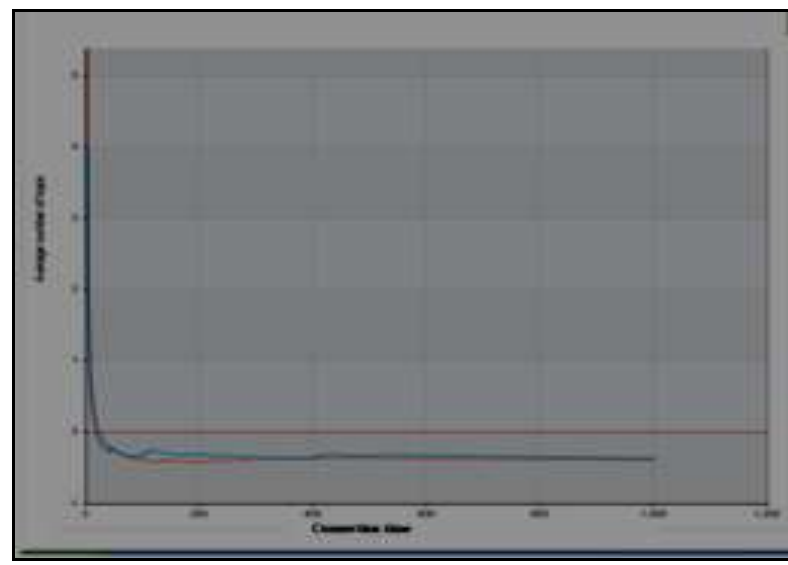

Fig 4 Average number of hops vs connection time

3) At third simulation in ant colony algorithm

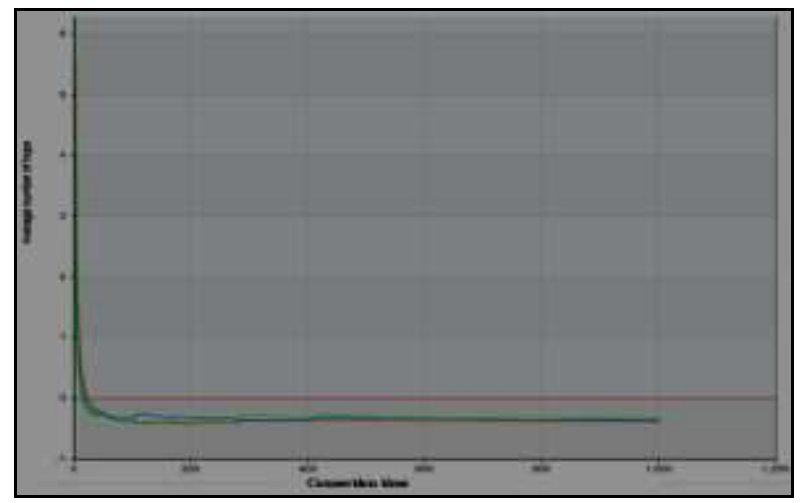

Fig 5 Average number of hops Vs connection time

\section{B. Modified ant algorithm}

The probability of some paths that are likely to be selected becomes low gradually when the algorithm is implemented for a certain period. In order to increase the possibility of exploring these paths, choose the path with highest probability. Initially select the path and visit all the nodes from source to destination and then select the good path after that select the next path and visit all the nodes and select the best path from all the good paths. In modified algorithm number of hops will be less.

\section{1) At first simulation in modified ant colony algorithm}

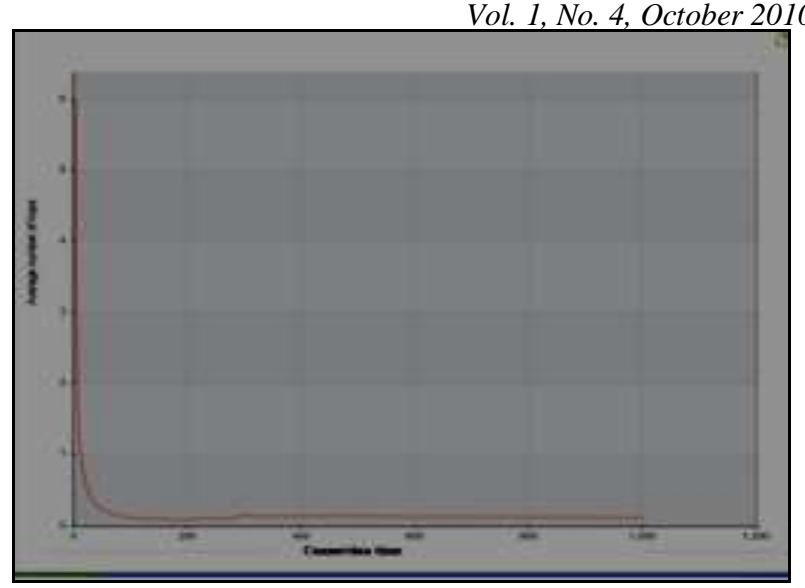

Fig 6 Average number of hops vs Connection time

Fig 6 shows the average number of hops on connection time with the modification of the probability in ant algorithm and number of hops will change according to the updation of the probability in 100 nodes it also shows the comparison between first simulation, second simulation and third simulation.

2) At second simulation in modified ant colony algorithm

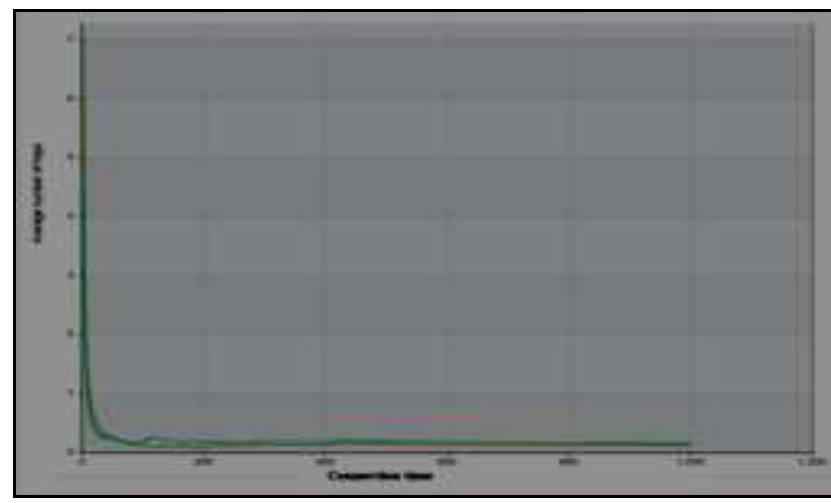

Fig 7 connection time vs average number of hops in modified algorithm for second simulation

3) At third simulation in modified ant colony algorithm

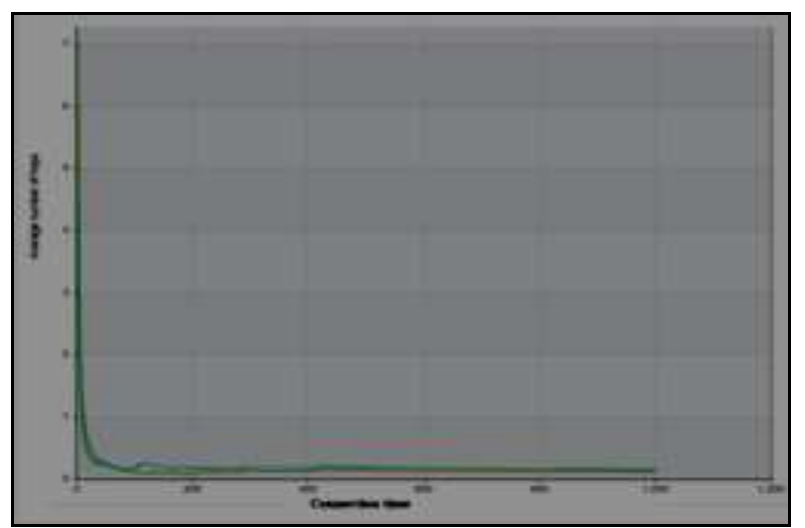

Fig 8 Average no of hops Vs connection time 
Fig 8 shows the average number of hops on connection time with the modification of the probability in ant algorithm and number of hops will change in 100 nodes it also shows the comparison between first simulation, second simulation and third simulation.

\section{Result}

\section{A. Comparison of Ant Algorithm and modified Ant Algorithm}

TABLE I

\begin{tabular}{|l|l|l|}
\hline \multirow{2}{*}{$\begin{array}{l}\text { S.N } \\
\text { o }\end{array}$} & \multicolumn{2}{|c|}{ Comparison of ant and modified ant algorithm } \\
\cline { 2 - 3 } & Ant algorithm & Modified ant algorithm \\
\hline 1 & $\begin{array}{l}\text { Ant will traverse only on } \\
\text { single path whose nodes } \\
\text { probability will be high } \\
\text { based on above formula. }\end{array}$ & $\begin{array}{l}\text { Ant will traverse on all } \\
\text { paths and then select the } \\
\text { best path from the good } \\
\text { paths. }\end{array}$ \\
\hline 2 & $\begin{array}{l}\text { With this algorithm we find } \\
\text { the good path not the best } \\
\text { path. }\end{array}$ & $\begin{array}{l}\text { This algorithm finds the } \\
\text { best path from the good } \\
\text { paths. }\end{array}$ \\
\hline 3 & $\begin{array}{l}\text { In this average number of } \\
\text { hops will be more as } \\
\text { compared to modified ACO. }\end{array}$ & $\begin{array}{l}\text { In this number of hops } \\
\text { will be less, and as } \\
\text { number of hops will be } \\
\text { less then traversing path } \\
\text { will be less. }\end{array}$ \\
\hline
\end{tabular}

B. Performance Comparison of Ant Algorithm and modified Ant Algorithm

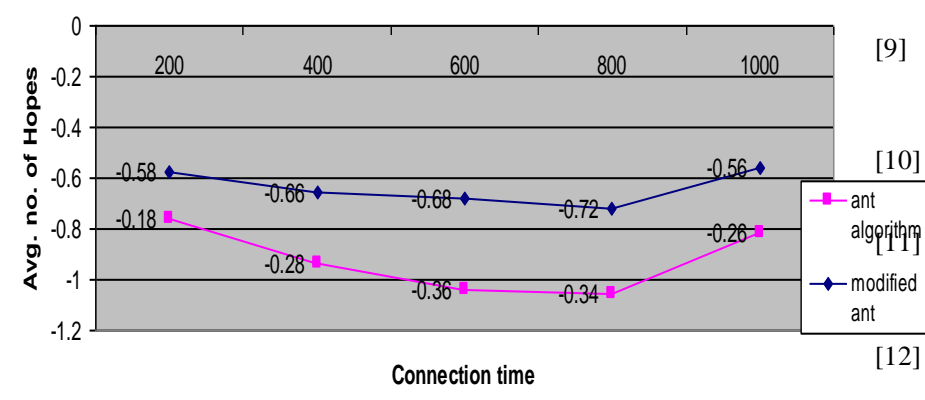

Fig. 9 Comparison between ant and modified ant algorithm

Fig 9 shows the comparison between ant algorithm and modified algorithm. As per the graph it is clearly visible that the modified ant algorithm is much better that ant algorithm.

This graph shows that the difference between ant algorithm and modified ant algorithm.

\section{CONCLUSIONS}

Modified ant algorithm explained in this paper solves the problem with a best solution that the other algorithms are easy to fall into the local optimal solution. As it takes probability into consideration the number of hops will be less in modified algorithm as compare to the simple ant algorithm. The result of simulation the experimental simulation shows that the quality and of the improved algorithm is superior to the traditional algorithms, and the improved algorithm solution is closed to the optimal solution. Therefore, the improved algorithm is an efficient way to solve the TSP as well as provide a new idea to solve other typical problems as well.

\section{ACKNOWLEDGMENT}

The author is grateful to the Department of CSE, Lingayas University, which has provided the systems to carry out my experimentation.

\section{REFERENCES}

[2] Hassin, R.; Rubinstein, S. (2000), "Better approximations for max TSP", Information Processing Letters 75: 181-186,

[3] Kaplan, H.; Lewenstein, L.; Shafrir, N.; Sviridenko, M. (2004), "Approximation Algorithms for Asymmetric TSP by Decomposing Directed Regular Multigraphs", In Proc. 44th IEEE Symp. on Foundations of Comput. Sci., pp. 56-65

[4] PELLEGRINI Paola, FAVARETTO Daniela, MORETTI Elena, "On MAX-MIN Ant System's Parameters," 5th International Workshop, ANTS2006, Brussels, Belgium, September 2006

[5] Marco Dorigo, Maniezzo, Colorni, "positive feedback as a search strategy,” Technical Report, pp. 91-106, 1991.

[6] Marco Dorigo, Colorni. "The Ant System: Optimization by a colony of cooperating agents," Systems, Man, and Cybernetics, Part B, IEEE Transactions on System, vol 26. pp.29-41, Feb 1996.

[7] Cerny.V, "A Thermodynamical Approach to the Travelling Salesman Problem: An Efficient Simulation Algorithm," J.Optimization Theory and Appl. 45(1985), pp.41-51.

[8] G. Di Caro, Vasilakos T., Ant -SELA: Ant -agents and stochastic automata learn adaptive routing tables for QoS routing in ATM networks , ANTS'2000 - From Ant Colonies to Artificial Ants: Second International Workshop on Ant Algorithms, Brussels, Belgium, September 2000.

[9] L.F. Escudero, An inexact algorithm for the sequential ordering problem. European Journal of Operational Research 37 (1988), 232253.

[10] W.J. Gutjahr, A graph-based Ant System and its convergence. Future Generation Computer Systems. 16, 873 - 888, 2000.

PELLEGRINI Paola, FAVARETTO Daniela, MORETTI Elena, "On MAX-MIN Ant System's Parameters," 5th International Workshop, ANTS2006, Brussels, Belgium, September 2006.

[12] Camelia-Mihaela Pintea, D.Dumitrescu. Improving ant systems using a local updating rule[C]. Proceedings of the Seventh International Symposium on Symbolic and Numeric Algorithms for Scientific Computing, 2005, 149 159.

[13] M.Dorigo, T. Stützle, Ant Colony Optimization[M]. MIT Press, Cambridge, MA,2004. P328.

[14] Applegate, D. L.; Bixby, R. M.; Chvátal, V.; Cook (2006), The Traveling Salesman Problem

[15] Bellman, R. (1960), "Combinatorial Processes and Dynamic Programming", in Bellman, R., Hall, M., Jr. (eds.), Combinatorial Analysis, Proceedings of Symposia in Applied Mathematics 10, American Mathematical Society, pp. 217-249

[16] Bellman, R. (1962), "Dynamic Programming Treatment of the Travelling Salesman Problem", J. Assoc. Comput. Mach. 9: 61-63.

[17] Christofides, N. (1976), "Worst-case analysis of a new heuristic for the travelling salesman problem", Technical Report 388, Graduate School of Industrial Administration, Carnegie-Mellon University, PittsburghM. Shell. (2002) IEEEtran homepage on CTAN. [Online].

[18] Information Processing Letters 82(3): 145-153 (2002).

[19] L.M. Gambardella and M. Dorigo, An ant colony system hybridized with a new local search for the sequential ordering problem, INFORMS Journal on Computing 12(2000), no. 3, 237--255. 
(IJACSA) International Journal of Advanced Computer Science and Applications,

[20] L.M. Gambardella and M. Dorigo, Ant -Q: a reinforcement learning approach to the travelling salesman problem, Proceedings of the Twelfth International Conference on Machine Learning, M L-95, Palo Alto, CA, Morgan Kaufmann, Palo Alto, California, USA, 1995.

[21] L.M Gambardella and M. Dorigo M, Solving Symmetric and Asymmetric TSPs by Ant Colonies, Proceedings of the IEEE Conference on Evolutionary Computation, ICEC96, Nagoya, Japan, May 20-22, 1996, pp. 622-627. 